\title{
Legislative and ethical aspects of introducing new technologies in medical care for senior citizens in developed countries
}

This article was published in the following Dove Press journal:

Clinical Interventions in Aging

21 July 2016

Number of times this article has been viewed

\author{
Jaroslav Kacetl' \\ Petra Maresova ${ }^{2}$ \\ 'Department of Applied Linguistics, \\ ${ }^{2}$ Department of Economics, Faculty \\ of Informatics and Management, \\ University of Hradec Kralove, Hradec \\ Kralove, Czech Republic
}

\begin{abstract}
Introduction: The majority of developed countries are currently experiencing demographic aging. The most frequently expressed concerns related to the changing age structure are the increased costs of social and medical care, a lack of labor force in the job market, and financial sustainability of the pension system. These concerns are often based on the pessimistic view of population aging. This view understands aging as a prolonged period of illness and suffering. On the other hand, optimists believe that a longer life span is a result of increased quality of life and better health care. The quality of life may be improved not only by medicaments, but also by rapidly developing area of medical devices, which allow better care for seniors in many areas.
\end{abstract}

Aim: This contribution aims to assess the legislative environment and ethical questions related to the use of medical devices, especially medical devices, in medical care for senior citizens.

Methods: The methods used in this study are literature reviews of legislative and ethical environment in the European Union (EU) and the US.

Results: Main findings of this study result from assessing the state of medical device regulations in Europe and the US. Namely, the US regulation seems to be better arranged, which is probably due to the fact that there is only one responsible body - the US Food and Drug Administration, which is responsible for all medical device regulations. On the other hand, in the EU, talks about new legislation are led by ministers from all the EU member states and it may take a long time before all the EU countries come to an agreement.

Keywords: senior citizen, medical devices, legislation, ethical aspects

\section{Introduction}

The majority of developed countries are currently experiencing demographic aging. ${ }^{1,2}$ The problem of its impacts was perceived as early as in the mid-twentieth century. However, it is becoming much more topical nowadays. National positive aging strategies are being established, retirement reforms implemented, and various research done. The most frequently expressed concerns related to the changing age structure are the increased costs of social and medical care, a lack of labor force in the job market, and financial sustainability of the pension system. These concerns are often based on the pessimistic view of population aging. This view understands aging as a prolonged period of illness and suffering.

On the other hand, optimists believe that a longer life span is a result of increased quality of life and better health care. The quality of life may be improved not only by medicaments, but also by rapidly developing area of medical devices, which allow better care for seniors in many areas. Positive effects of medical devices are discussed
Correspondence: Petra Maresova Department of Economics, Faculty of Informatics and Management, University of Hradec Kralove, Rokitanskeho 62, Hradec Kralove 3, 50003, Czech Republic Tel +420737927 745 Email petra.maresova@uhk.cz 
by various authors. McKee et $\mathrm{al}^{3}$ mention that patients using medical devices relish increased independence, better physical and mental health, and improved quality of life. Gregory ${ }^{4}$ emphasize that those who utilize medical devices enjoy better health and more safety, and Gustafson et $\mathrm{al}^{5}$ maintain that medical devices help lower health care costs among older adults. For instance, the new term telemedicine, meaning distance technologies of patient monitoring, represents these new possibilities. Distant medical monitoring (DMM) saves both financial and human resources and shortens emergency response times. Mobile systems of DMM can help both doctors and patients by providing an instant access to medical information, particularly in emergency. ${ }^{6}$ Telemedicine offers another interesting benefit, namely, monitoring of patients based on multifunctional wearable sensors, which help patients live more healthy life. Clinical assessments have shown that wearable sensors do not limit the normal daily activities of the patients. Reiss and Stricker ${ }^{7}$ have shown that on average up to $43 \%$ of patients avoid visiting the doctor in person. ${ }^{8}$

The development of medical devices may significantly improve care for senior citizens by improving their safety as well as quality of life. ${ }^{9}$ Legislative environment and implementing new medical devices are two closely intertwined areas in every country. Legislation may either boost or hinder the launch of a new product into the market by specifying conditions of its utilization. Laws and regulations are therefore the key factors in the expansion of the medical device market. With respect to medical devices, another significant area is ethics. There exist a lot of opportunities for ethical considerations in this field, including the disturbance of senior citizens' privacy as a result of utilizing various types of monitoring devices. It also seems to be quite important to know potential users' attitudes to using particular types of new medical devices.

For this reason, the aim of this contribution is to assess the legislative environment and ethical questions related to using medical devices in medical care for senior citizens. Attention is paid mainly to the European Union (EU) and the US as these countries face problems with aging population more acutely than most other countries and have to look for solutions to problems caused by their demographic development. Thus, care for senior citizens is becoming one of the key issues for both the EU and the US.

The term "medical devices" is understood in this study as it has been specified by the World Health Organization (WHO): any instrument, apparatus, implement, machine, appliance, implant, reagent for in vitro use, software, material or other similar or related article, intended by the manufacturer to be used, alone or in combination, for human beings, for one or more of the specific medical purpose(s) of: ${ }^{10}$
- diagnosis, prevention, monitoring, treatment, or alleviation of disease,

- diagnosis, monitoring, treatment, alleviation of, or compensation for an injury,

- investigation, replacement, modification, or support of the anatomy or of a physiological process,

- supporting or sustaining life,

- control of conception,

- disinfection of medical devices

- providing information by means of in vitro examination of specimens derived from the human body;

and does not achieve its primary intended action by pharmacological, immunological, or metabolic means, in or on the human body, but which may be assisted in its intended function by such means.

As this definition is broad, it is impossible to cover all legislative and ethical aspects linked to medical devices in this study. The focus has therefore been narrowed to European and American legislation and to ethical aspects related to elder care in areas such as developing new medical devices and their utilization in treatment, monitoring the elderly's behavior and health, or alleviating their suffering by assisting them in their daily tasks. All this with respect to patients needs. ${ }^{11}$

\section{Methods}

Legislative environment and ethical considerations, which are closely related to medical device utilization in the field of medical care for senior citizens in both the EU and the US, were assessed by means of literature review, using the official websites of legislative bodies responsible for American and European medical device regulations, respectively. Other related studies were analyzed too. Also, texts from ScienceDirect database were studied. The search was done for keywords "medical devices AND ethics" and "medical devices AND legislative environment" for the period from 2010 to 2015. The list of generated articles was studied and $\sim 20$ most relevant texts were determined, accessed, and studied.

\section{Legislative environment in the EU and the US \\ Medical device regulation in the US}

In the US, the ultimate authority accountable for regulating firms involved in local medical device business is the US Food and Drug Administration (US FDA), which is one of the operating divisions of the US Department of Health \& Human Services. ${ }^{12-14}$ The FDA monitors various issues that concern a wide range of medical devices from simple to much more complex ones. 
The US FDA is responsible for the Code of Federal Regulations, Title 21, which contains rules for food and drugs. Medical device regulation can be found in Chapter I - Food and Drug Administration, Department of Health and Human Services. ${ }^{15}$ It covers, among other things, medical device labeling and reporting, premarket approval, postmarket surveillance, or unique device identification. There are rules applicable to various types of devices, including, for instance, cardiovascular; dental; or ear, nose, and throat devices. The website Registrar Corp ${ }^{12}$ lists FDA regulations as follows:

U.S. FDA Medical Device Regulation: 21 C.F.R. Part 801 et seq., section 814.9; UDI-GUDID Regulations: 78 Fed. Reg. 58785, 58785-58828; U.S. FDA MAF Regulation:

21 C.F.R. section 814.9.; U.S. FDA Color Additives: 21 C.F.R. Parts 73, 74, 80.

The FDA annually receives hundreds of thousands of reports of malfunctions, injuries, or even deaths having to do with the use of medical devices. This practice is called Medical Device Reporting. ${ }^{13}$ There are both mandatory reporters, including manufacturers or importers, who have to report any device-related problems, and voluntary ones, including patients or caregivers, who also may inform FDA regarding adverse events that might have been caused by the use of medical devices. The FDA then alerts all the involved parties as well as the public in case of any risk involved in using a particular medical device.

Based on receiving Medical Device Reporting, the FDA monthly updates a large database of reports, called the Manufacturer and User Facility Device Experience (MAUDE). It is used to monitor device performance, detect potential device-related safety issues, and contribute to benefit-risk assessments of these products. Although it is an invaluable source of information, MAUDE is in no way impeccable. Its limitations include, among other problems, potentially flawed reporting, which may be inaccurate, biased, or insufficient.

Another similar online reporting platform, launched in 2002 by the Center for Devices and Radiological Health (CDRH), is the Medical Product Safety Network (MedSun). It facilitates cooperation and communication between the FDA and the clinical community. It helps identify, understand, and solve problems with the use of medical devices. ${ }^{13}$

Information gathered about adverse events and other problems potentially caused by medical devices are used by the $\mathrm{CDRH}$ to assess and prevent regulatory science risks. A document called CDRH Regulatory Science Priorities (FY2016) has identified ten priorities for the year $2016 .{ }^{16}$ The document says that $\mathrm{CDRH}$ "is responsible for assuring the safety, effectiveness, performance and quality of medical devices and radiationemitting products used to treat, prevent, and diagnose disease." ${ }^{\prime 16}$ Besides the key terms of safety, effectiveness, performance, and quality, the document also mentions the importance of facilitating medical device innovation and development; using state-of-the-art technologies; ensuring good decision making in the areas of premarket evaluation, postmarket surveillance, compliance, and education; ${ }^{16}$ and improving the knowledge of the benefit-risk profile of all devices.

\section{Medical device regulation in the EU}

In Europe, the European Commission is the authority in the area of medical devices. As such, it is responsible for the corresponding legislation. There are currently three directives that create the legal framework for regulating medical devices within the EU. Namely, Council Directive 90/385/ EEC on Active Implantable Medical Devices (AIMDD) implemented in 1990, Council Directive 93/42/EEC on Medical Device Directive implemented in 1993, and Council Directive 98/79/EC on In Vitro Diagnostic Medical Devices (IVDMD) implemented in 1998. These directives have been amended several times. ${ }^{17}$ In 2015, a general approach on a new medical devices package was agreed on by the ministers of the EU countries. The preliminary talks had taken almost 3 years. It is considered as a major step toward implementing new medical devices regulations, which should lead to an increased level of safety for EU citizens using medical devices. There is, however, still ambiguity as to what the new EU's medical device directives will look like and when their final version will come into effect. ${ }^{18}$

The medical devices sector is becoming increasingly important in the EU. The European Commission understands the role of medical devices as essential to the health care of European citizens. ${ }^{17}$ Medical devices cover a wide range of products important to the health and quality of life and in the diagnosis, prevention, monitoring, and treatment of diseases as well as for improving the quality of life of people with disabilities. ${ }^{17}$ With 575,000 employees working for $\sim 25,000$ (95\%) small- and medium-sized enterprises, the medical devices sector is a major employer in Europe with total sales reaching $€ 100$ billion. The most challenging issues this sector faces are health inequalities, an aging society, research and development in medical devices sector, emerging e-health technologies, patients' better access to medical devices, the balance between patients' needs, and financial sustainability.

There are several bodies that are consulted when a problem arises; among them are the Scientific Committee on Emerging 
and Newly Identified Health Risks (SCENIHR) and the New and Emerging Technologies Working Group (NET), which aim to follow all new and emerging technologies and their impact in the field of medical devices at the EU Commission level. ${ }^{19}$ European Databank on Medical Devices (Eudamed) provides fast access to relevant information and thus aims to improve market surveillance and transparency in this area.

Problem areas include reprocessing medical devices or counterfeiting of health products. The latter is a serious issue because non-authentic products may cause adverse effects with dire health as well as economic implications. Therefore, it is important to prevent counterfeit medical devices from leaking into the EU market. The Unique Device Identification (UDI) should contribute to more efficient traceability of medical devices by identifying both the device and its producer. The document titled UDI Guidance - Unique Device Identification (UDI) of Medical Devices was adopted in December 2013. ${ }^{20}$

\section{Ethical aspects}

There are a lot of ethical challenges in a fast growing area like the medical device industry. Sadly, even the best ideas and inventions may have potentially dangerous and bad unexpected results. It is virtually impossible to prevent all of them from happening. Moreover, even more alarmingly, ethical considerations may clash with business ones. For example, according to Holtzman et al, ${ }^{18}$ major reasons for slowing in the US medical device industry are, among other things, a shift to less risky, more evolutionary research, and burdensome regulatory environment enforced by FDA. Citron $^{21}$ insists that it is generally slower and more expensive to receive FDA's approval in the US than in Europe and other developed markets. The same author adds that the EU countries rely more on doctors' responsibility to assess medical devices than on research and development (R\&D) and producers. It seems vague and unclear as who should have the final word and be ultimately responsible for decision-making processes on a global scale.

Nevertheless, from the ethical point of view it is a good practice to do one's best to keep potential issues caused by new medical devices to a minimum. To do that, it is necessary both to keep track of any problems that have already occurred and try to logically anticipate potential consequences of something that does not exist yet.

The first way is reflected in creating and maintaining the aforementioned medical device report databases or reporting platforms such as MAUDE or MedSun in the US and Eudamed in Europe, or expert bodies such as SCENIHR or NET that are consulted when a risk or a problem with new technologies arises. These institutions constitute an opportunity to immediately respond to any ethical problems, malfunction, injuries, or deaths potentially caused by the use of medical devices. Unfortunately, there are still many flaws in these control mechanisms, and it remains to be determined how to efficiently use the collected information in practice.

The latter way is based on sharp thinking and wide experience of experts who feel responsible to warn others about potential dangers they can see. Truly, ethical problems in the field of medical devices are galore, which include fast technological development, diverse ethical threats posed by different medical devices as well as by various areas of medical science, keeping a clear distinction between humans and machines, societal consent, human and patients' rights, and patient-centered views of medical devices. According to Meskó, ${ }^{22}$ medical devices may bring both relief to patients and potential dangers that range from discomfort to death, even to potential homicide. The following text lists several examples of ethical threats in order to give an idea of what problems are - or might be - identified and dealt with.

Medical devices are intended to provide patients with considerable benefits. Nonetheless, they may cause them serious harm. Logothetidis ${ }^{23}$ has warned that it is indispensable to forecast and address unexpected ethical - as well as environmental and health - consequences of new technologies used in medicine. This poses a real problem as technologies develop very fast, which makes it virtually impossible to anticipate all potential threats involved with them.

The US FDA has released an update on what medical devices might be developed and launched into the market within the next 10 years. ${ }^{24}$ For instance, the FDA expects the following ones: integrated electronic patient medical records, computer-assisted diagnostic systems, internet-based medical device systems, virtual reality systems for immersive training and other applications, robotic products and systems, neurosensory devices, minimally invasive therapeutic products, self-care products, and patient monitoring systems.

Moreover, different areas of human interest bring different ethical problems. Ethical considerations involved in the area of medical devices differ from those present in other areas of medical science. For instance, according to Citron, ${ }^{21}$ medical device R\&D and product application have a number of relatively unique aspects that distinguish them from other technologies such as pharmaceuticals; especially, he claims that $R \& D$ practices and ethical considerations related to medical devices are unique. Therefore, other ethical 
considerations should apply to medical devices than to any other branch of medical science.

It is by no means an easy task to anticipate all potential threats that medical devices may cause. Although some potential problems might be spotted in advance, either by experts in ethics or medical devices, or by potential users of medical devices, others may appear only during the use of the medical device. Therefore, Sharley and Sharley ${ }^{25}$ have highlighted the necessity of detailed consultations with elderly people to make sure that medical devices improve their lives. $\mathrm{Ng}^{26}$ emphasized that rapid development of medical devices and their fast integration into medical practice make it impossible to anticipate their potentially harmful effects; he has also added that recent innovations in this area further blur the distinction between human beings and machines, which may lead to redefining the concept of the self.

There are a lot of other ethical concerns in this area. Meskó $^{22}$ lists hacking of medical devices as the most important ethical issue of future medicine and adds that it can be expected that soon people will be murdered in this way. Another trouble is potential discrepancy between societal ethical norms and/or religious beliefs on the one hand and latest medical innovations on the other hand. ${ }^{21}$ Ikonen and Kaasinen ${ }^{27}$ have also emphasized that the rules of conduct result from mutual interaction among the law, ethics, and religion. Researchers therefore also have to take into account possible societal responses to their projects. What is more, these responses may differ from country to country (or culture to culture) from encouraging to lukewarm to negative and angry ones.

Kriewall ${ }^{28}$ has suggested that it is desirable for medical devices to offer patients maximum flexibility and safety for reasonable price. Chao et al ${ }^{29}$ call it a patient-centered approach. Chao et al ${ }^{29}$ call for a patient-centred approach, which they illustrate on the Stanford Biodesign Program. This program is meant to help students and postgraduates in the fields of medicine, engineering, law, and business start projects aiming at producing new and innovative medical devices. The basic principles are as follows: doing patients good, not doing any harm, respecting other people's rights, and deciding fairly among competing interests.

Friedman and $\mathrm{Kahn}^{30,31}$ have determined 12 specific human values that are implicated in the human-computer interaction community: human welfare, ownership and property, privacy, freedom from bias, universal usability, trust, autonomy, informed consent, accountability, identity, calmness, and environmental sustainability. However, they are well aware of the pitfalls linked to making similar lists. They confess that their list is not - and, as they suggest, perhaps no list can be - comprehensive. Moreover, they admit that not all the listed values are distinct from one another. It is therefore no surprise that different authors offer their own lists.

Mittelstadt et a ${ }^{32}$ have conducted a literature review trying to identify ethical themes present in the current literature about medical devices, even though they use the term Personal Health Monitoring (PHM) technologies. They define PHM as "any electronic device or system that monitors a health-related aspect of a person's life on a constant basis outside of a hospital setting." The authors have identified eight ethical themes: 1) privacy, either personal privacy or data privacy, as information of this kind may be misused; 2) visibility, which refers to how noticeable tracking devices are and raises an issue of covert surveillance; 3) medicalization of the home environment; 4) social isolation as PHM devices may make visits of human caregivers less necessary; however, less frequent social interaction may be substituted with social networking features built into PHM devices; 5) autonomy, or the right to make decisions for oneself, as PHM devices may reduce patients' autonomy and alter their daily routine; 6) balancing of conflicting values, like those of freedom and safety, within a family or in doctor-patient or family-patient relationships, which can be done by means of an individualized approach to using PHM technologies; 7) necessity of ethical discussions during the development and implementation of PHM technologies; and 8) the impact of PHM on medical personnel. Even though all these potential ethical threats have been mentioned in articles reviewed by Mittelstadt et al, ${ }^{32}$ the authors point out that a few of the studied texts feature in-depth discussions of ethics.

Ikonen and Kaasinen ${ }^{27}$ aim to provide guidance on ethical issues linked to ambient-assisted living (AAL), namely, mobile-centered ambient intelligence, which may be applied in health care and can help senior citizens as well as others in need to live independently at home. Memon et $\mathrm{al}^{33}$ define AAL as "an emerging multi-disciplinary field aiming at exploiting information and communication technologies in personal healthcare and telehealth systems for countering the effects of growing elderly population," which means AAL belongs to - or is at least closely linked to - medical devices as it is defined by WHO. ${ }^{10}$ Ikonen and Kaasinen ${ }^{27}$ have listed six ethical principles that they deem crucial in the area of AAL. First, privacy means control over one's personal information and space. The second principle, autonomy, refers to the right to decide how to use technology. Integrity and dignity imply that individuals should always be more respected than technology. Reliability hints at the 
fact that no technology should endanger its user's physical or mental health. E-inclusion suggests that even physically and mentally disadvantaged must have access to technologies. The last principle, role of technology in the society, signifies that technology should increase the quality of life without harming anybody.

According to Ikonen and Kaasinen, ${ }^{27}$ no ethical guidelines can prevent all risks. Therefore, it is necessary to continue to refine these guidelines and to do ethical assessment during the whole product life cycle. This assessment ought to be done in two ways. First, it is important to gather information from interviews with users and from their evaluations. Second, experts on ethics should assess all potential risk.

Sharley and Sharley ${ }^{25}$ have mentioned the use of robots in elderly care. Robots, which can also be considered medical devices according to the WHO definition, ${ }^{10}$ are used in three ways - to assist the elderly, to monitor their behavior and health, and to provide companionship. The authors have identified, raised, and discussed the following six potential ethical concerns: 1) the potential reduction in the amount of human contact, 2) an increase in the feelings of objectification and loss of control, 3) a loss of privacy, 4) a loss of personal liberty, 5) deception and infantilization, and 6) the circumstances in which elderly people should be allowed to control robots. These ethical concerns are related to human rights and shared human values established in widely accepted documents such as the Charter of the United Nations ${ }^{35}$ and the Universal Declaration of Human Rights, ${ }^{35}$ and the authors aim to identify both the possible advantages and potential risks of different forms of robot use. They conclude that we should be concerned about the use of robots for elderly care and suggest three methods to mitigate the potentially harmful effects. First, they propose developing guidelines or even legislation about robot use. Second, they recommend basing these guidelines on carefully controlled studies and consultations with the elderly and encouraging the development of customized solutions. Third, they believe it is advisable to make robot developers take a value-sensitive design approach and encourage them to create robots that would prevent themselves from offending against the identified ethical concerns.

\section{Discussion}

Technology is allowing us to measure critical health parameters in convenient and inexpensive ways. Tiny, wearable sensors collect data without interfering with daily life, enabling better-informed and more quantified decision making. ${ }^{6}$ Electronic clothing paired with sensors is an outlet used to collect such data. Home health care services and innovative technology are expected to allow for doctor-patient connectivity, ${ }^{33}$ which have not been possible, thus saving both lives and money. Patient monitoring before, during, and after a procedure can now include autonomous robots. ${ }^{25}$

On comparing the medical device regulation in Europe with that in the US, it has been found that the US regulation seems to be better arranged, which is probably due to the fact that there is only one responsible body, namely, the US FDA, which is accountable for all medical device regulations. On the other hand, in the EU, talks about new legislation are led by ministers from all the EU member states and it may take a long time before all the EU countries come to an agreement. For instance, the preliminary talks about the new EU medical device legislation to be implemented in the near future took 3 years. Although the US medical device regulation seems to be continually revised, the EU regulatory framework is quite obsolete with three core directives from 1990, 1993, and 1998. Nonetheless, a new medical device package is awaited in the near future.

A lot of ethical challenges lay in store for medical device industry. According to Keselman et al, ${ }^{36}$ many medical devices that are currently in the market are suboptimal for human use. Ethics is rarely mentioned in both the US and EU medical device regulations. For instance, current EU directives only mention that national regulations relating to ethics continue to apply and they refer to the Declaration of Helsinki, ${ }^{37}$ which is the World Medical Association's statement on ethical principles for medical research involving human subjects.

On the other hand, it is good news that there are institutions like MAUDE, MedSun, Eudamed, SCENIHR, and NET that record and try to solve any problems linked to using medical devices. However, these institutions cannot efficiently treat all ethical issues. It is therefore advisable to look for ways on how to use the collected information and experience in order to prevent the same bad things from happening again.

Apart from the already existing issues, there are also some potential future dangers; some of them are really serious, including potential homicide. According to Meskó, ${ }^{22}$ it remains to be seen whether they will become real dangers and how they will be dealt with. Meskó ${ }^{22}$ presumes that mankind may expect fundamental changes in health care. Currently, diagnostic procedures are shifting toward portable devices and can be performed from home. Medical mobile applica- 
tions will be patient customized. Meskó22 believes that "the smartphone will be the hub of the future of medicine, serving as a health-medical dashboard."

Yet another issue rises from the fact that there is certain discrepancy between discussing ethical concerns in scientific texts and their practical solving. As Mittelstadt et $\mathrm{al}^{32}$ pointed out, a lot of academic articles mention, or even list various ethical issues, but few of these papers offer in-depth discussions of them, or even practical solutions to these problems. It would be ideal if there existed a globally or internationally accepted problem-solving procedure for the area of medical devices. Nevertheless, as it has been discussed, the US medical device regulation differs from that of the EU. Furthermore, there are various national views within the EU and finding a compromise usually means protracted negotiations as in the case of the new EU's medical devices package. ${ }^{17}$

This paper identified several important strategies on how to ensure that medical devices will help their users without causing any unnecessary dangers to them; keeping records of risks is one of them. Then, the paper showed that some experts (eg Meskó ${ }^{22}$ ) identify and anticipate potential threats linked to using medical devices. It is also vital to gather information from detailed consultations with medical device users as well as caregivers. Collecting this kind of data is an opportunity for continual research in this area.

The aforementioned sources of information constitute an empirical basis for further discussions about new medical device legislation. Another basis can be seen in internationally accepted documents on human rights like the Charter of the United Nations, ${ }^{34}$ the Universal Declaration of Human Rights, ${ }^{35}$ or the Declaration of Helsinki. ${ }^{38}$

All in all, it seems that all attempts to make medical devices safe for use go down to what is called a patientcentered approach, ${ }^{29}$ which may be understood as applying shared human values on assessing practical utilization of medical devices. The most frequently mentioned values are privacy $^{25,27,30,32}$ and autonomy. ${ }^{27,30,32}$ Arguably, the ultimate source of all these shared values is human dignity.

\section{Acknowledgment}

This research was supported by the project Excellence (University of Hradec Kralove, Faculty of Informatics and Management) and the internal research Economic and Managerial Aspects in Biomedicine.

\section{Disclosure}

The authors report no conflicts of interest in this work.

\section{References}

1. Maresova P, Mohelska H, Kuca K. Economics aspects of ageing population. Procedia Econ Finance. 2015;23:534-538.

2. Maresova P, Kacetl J, Stemberková R, Kuca K. Care for Czechś Ageing Population. Proceedings of the 19th International Conference Current Trends in Public Sector Research, Brno, Czech Republic. 2015: 250-258. Brno, Czech Republic.

3. Mckee K, Matlabi H, Parker SG. Older people's quality of life and role of home-based technology. Health Promot Perspect. 2012;2(1):1-8.

4. Horgas A, Abowd G. The impact of technology on living environments for older adults. In: National Research Council (US) Steering Committee for the Workshop on Technology for Adaptive Aging; Pew RW, Van Hemel SB, editors. Technology for Adaptive Aging. Washington (DC): National Academies Press (US); 2004:9.

5. Gustafson DH Sr, McTavish F, Gustafson DH Jr, et al. The effect of an information and communication technology (ICT) on older adults' quality of life: study protocol for a randomized control trial. Trials. 2015; 16:191.

6. Baig MM, Gholamhosseini H. Smart health monitoring systems: an overview of design and modeling. J Med Syst. 2013;37(2):9898.

7. Reiss A, Stricker D. Aerobic activity monitoring: towards a long-term approach. Universal Access Information Society. 2014;13(1):101-114.

8. Wootton R, Bahaadinbeigy K, Hailey D. Estimating travel reduction associated with the use of telemedicine by patients and healthcare professionals: proposal for quantitative synthesis in a systematic review. $B M C$ Health Serv Res. 2011;11:185. Available from: http://bmchealthservres. biomedcentral.com/articles/10.1186/1472-6963-11-185. Accessed December 14, 2015.

9. Maresova P, Penhaker M, Selamat A, Kuca K. The potential of medical device industry in technological and economical context. Ther Clin Risk Manag. 2015;11:1505-1514.

10. WHO. Medical devices full definition. 2016. Available from: http://www.who.int/medical_devices/full_deffinition/en/. Accessed December 14, 2015.

11. Chao KZ, Riskin DJ, Krummel TM. A patient-centered, ethical approach to medical device innovation. Virtual Mentor. 2010;12(2):91-95.

12. Registrar Corp [homepage on the Internet]. U.S. FDA Medical Device Regulations. Available from: http://www.registrarcorp.com/fdamedical-device/registration/?lang=en. Accessed December 14, 2015.

13. FDA.gov [homepage on the Internet]. Medical devices. Available from: http://www.fda.gov/MedicalDevices/default.htm. Accessed December 14, 2015.

14. HHS.gov [homepage on the Internet]. About HHS, Department of Health \& Human Services. Available from: http://www.hhs.gov/about/ index.html. Accessed December 14, 2015.

15. GPO [homepage on the Internet]. Electronic Code of Federal Regulations. Available from: http://www.ecfr.gov/cgi-bin/text-idx?SID=9 97ee953cbebe663b8b9cc29,87c67076\&mc=true\&tpl=/ecfrbrowse/ Title21/21 chapterI.tpl. Accessed December 14, 2015.

16. Center for Devices and Radiological Health. Regulatory Science Priorities (FY2016). Center for Devices and Radiological Health; 2015:9. Available from: http://www.fda.gov/downloads/MedicalDevices/ ScienceandResearch/UCM467552.pdf. Accessed July 5, 2016.

17. European Commission (EC) [homepage on the Internet]. Medical devices. Available from: http://ec.europa.eu/growth/sectors/ medical-devices/index_en.htm. Accessed December 14, 2015.

18. Holtzman Y, Gorkhover G, Ganz M. The U.S. Medical Device Industry: Strengths, Weaknesses, Opportunities, and Threats. MD+DI, Medical Device and Diagnostic Industry. 2015.

19. Obelis [homepage on the Internet]. Europe moves forward on new and emerging technologies. Available from: http://obelismedical.net/ europe-moves-forward-on-new-and-emerging-technologies. Accessed December 14, 2015.

20. IMDRF. UDI Guidance Unique Device Identification (UDI) of Medical Devices. IMDRF; 2013. Available from: http://www.imdrf.org/docs/ imdrf/final/technical/imdrf-tech-131209-udi-guidance-140901.pdf. Accessed November 13, 2015. 
21. Citron P. Ethics considerations for medical device R\&D. Prog Cardiovasc Dis. 2012;55(3):307-315.

22. Meskó B. The Guide to the Future of Medicine: Technology and the Human Touch. Berlin: WEbicina Kft; 2014:274.

23. Logothetidis $\mathrm{S}$. Nanotechnology in medicine: the medicine of tomorrow and nanomedicine. Hippokratia. 2006;10(1):7-21.

24. FDA. FDA forecasts medical device technology trends for next decade. 2011. Available from: http:/www.dicardiology.com/content/ fda-forecasts-medical-device-technology-trends-next-decade\#sthash. zawYs9DD.dpuf. Accessed November 14, 2015.

25. Sharkey A, Sharkey N. Granny and the robots: ethical issues in robot care for the elderly. Ethics and Information Technology. 2012; 14(1):27-40.

26. $\mathrm{Ng} \mathrm{T}$. Ethics in the age of medical device technologies. Virtual Mentor. 2007;9(2):83-85.

27. Ikonen V, Kaasinen E. Ethical Assessment in the Design of Ambient Assisted Living. Proceedings of Assisted Living Systems-Models, Architectures and Engineering Approaches, Schloss Dagstuhl, Germany, 2008:14-17.

28. Kriewall TJ. Ethics of medical device safety. J Long Term Eff Med Implants. 2008;18(2):167-174.

29. Chao KZ, Riskin DJ, Krummel TM. A patient-centered, ethical approach to medical device innovation. Virtual Mentor. 2010;12(2):91-95.

30. Friedman B, Kahn PH. Human Values, Ethics and Design. University of Washington; 2003:1177-1201.
31. Friedman B, Kahn PH Jr. Human agency and responsible computing: implications for computer system design. J Syst Softw. 1992:17(1): $7-14$.

32. Mittelstadt B, Fairweather NB, McBride N, Shaw M. The ethical implications of personal health monitoring. Int J Technoethics. 2014; 5(2):37-60.

33. Memon M, Wagner SR, Pedersen CF, Beevi FHA, Hansen FO. Ambient assisted living healthcare frameworks, platforms, standards, and quality attributes. Sensors. 2014;14:4312-4341.

34. United Nations. Charter of the United Nations. The Statute of the International Court of Justice. San Francisco, CA: United Nations; 1945.

35. United Nations. The Universal Declaration of Human Rights. Available from: http://www.un.org/en/universal-declaration-human-rights/index. html. Accessed December 14, 2015.

36. Keselman A, Tang X, Patel VL, Johnson TR, Zhang J. Institutional decision-making for medical device purchasing: evaluating patient safety. Stud Health Technol Inform. 2004;107(Pt 2):1357-1361.

37. Herman WA, Devey GB. Future Trends in Medical Device Technologies. The U.S. Food and Drug Administration.

38. World Medical Association. World Medical Association Declaration of Helsinki: ethical principles for medical research involving human subjects. J Postgrad Med. 2002;48(3):206-208.
Clinical Interventions in Aging

\section{Publish your work in this journal}

Clinical Interventions in Aging is an international, peer-reviewed journal focusing on evidence-based reports on the value or lack thereof of treatments intended to prevent or delay the onset of maladaptive correlates of aging in human beings. This journal is indexed on PubMed Central, MedLine,

\section{Dovepress}

CAS, Scopus and the Elsevier Bibliographic databases. The manuscript management system is completely online and includes a very quick and fair peer-review system, which is all easy to use. Visit http://www.dovepress. com/testimonials.php to read real quotes from published authors. 